\title{
No evidence for a parental inversion polymorphism predisposing to rearrangements at $22 q 11.2$ in the DiGeorge/Velocardiofacial syndrome
}

\author{
European Journal of Human Genetics (2002) 11, 109-111. doi:10.1038/sj.ejhg.5200930
}

By now it is well established that most, if not all, common microdeletion syndromes arise by unequal intra or interchromosomal recombination between intrachromosomal segmental DNA duplications (also called duplicons) flanking the microdeleted regions (reviewed by Lupski ${ }^{1}$ and $\mathrm{Ji}$ et $a l^{2}$ ). In addition to the flanking duplicons, recent observations suggest that the presence of heterozygous inversion polymorphisms between the duplicons may confer a higher susceptibility for generating microdeletions. Osborne et $a l^{3}$ demonstrated that an inversion polymorphism was present in one-third of parents-of-origin of probands with the $1.5 \mathrm{Mb}$ deletion at $7 \mathrm{q} 11.23$ causing Williams-Beuren syndrome (WBS). Equally, an inversion polymorphism on chromosome $8 p$ was present in all mothers whose offspring had a deletion of the corresponding gene cluster of the olfactory receptor. ${ }^{4,5}$ These observations raise the question whether parental inversion polymorphisms are a common phenomenon in other microdeletion syndromes as well. In this study we investigated whether an inversion polymorphism might exist within the 22q11.2 commonly deleted region.

The 22q11.2 microdeletion, Velocardiofacial syndrome (VCFS) is the cause of a variety of clinical syndromes including the DiGeorge anomaly, Sphrintzen syndrome and the conotruncal anomaly face syndrome. It is the most frequent deletion syndrome in Man, occurring at a frequency of $1: 4000$ live births. ${ }^{6}$ Most patients share a typical $3 \mathrm{Mb}$ hemizygous microdeletion encompassing almost 30 contiguous genes. Low-copy repeats of approximately $200 \mathrm{~kb}$ flank the commonly deleted region. This $200 \mathrm{~kb}$ region comprises a tandem set of genes and pseudogenes, flanked on either side by inverted sub-repeats. ${ }^{7-9}$ Since it has been demonstrated that the presence of inverted repeats can cause inversions, ${ }^{10,11}$ it seems feasible that the presence of these large $(>10 \mathrm{~kb})$ inverted repeats within the low copy repeats flanking the VCFS microdeletion region are able to induce inversions.

The parents of 18 patients with VCFS and 22q11.2 deletion (as determined by FISH) were screened for the presence of an inversion polymorphism within the $22 \mathrm{q} 11.2$ deletion region. Both parents were examined blind as to the parent-of-origin. The parent contributing the normal chromosome 22 can be considered as a random population sampling. Using combinations of probes in two-color interphase FISH experiments (with two probes from within the common deletion and one from outside), we determined the orientation of the 22q11.2 commonly deleted region relative to the flanking DNA.

Probes were constructed by isolating genomic DNA from cosmids and labelling it with fluorochromes. ${ }^{9}$ Cosmid 106E4 containing marker D22S427 centromeric of the common deletion was labeled both with Biotin $\left(\right.$ Roche $^{\mathrm{TM}}$ ) and Digoxigenin (Roche $\mathrm{CM}^{\mathrm{TM}}$ ) to produce a dual-colour yellow signal using the BioNick ${ }^{\mathrm{TM}}$ Labelling System kit (Life Technologies). Cosmid 87H3 was labelled with digoxigenin (red) and cosmid 68A1 (marker D22S788) more telomeric within the deletion labelled with biotin (green) (Figure 1a). FISH was performed as described. ${ }^{3}$ Hybridisation was performed on interphase chromosomes in lymphocytes obtained from a peripheral blood sample. Cells airdried on slides were pretreated with pepsin, followed by denaturing in a $70 \%$ formamide $/ 2 \times \mathrm{SSC}$ solution at $72^{\circ} \mathrm{C}$ and then dehydration with ethanol. Signals were detected using TRITC labelled anti-digoxigenin and fluorescein labelled avidin (Roche). The cells were counterstained with DAPI. Nuclei where the three probes occurred close to each other and in a linear arrangement to exclude possible misalignment were examined. At least 100 interphases were scored per parent.

An average of 91\% (range 79-96\%) of all evaluated cells had the three probes in the expected order (Figure 1b). This result makes us conclude that none of the parents had an inversion polymorphism, which makes it unlikely that inversion polymorphisms within 22q11.2 microdeletion region are a major susceptibility factor in the generation of 22q11 microdeletions.

Current models describing the deletion causing mechanism predict an equal occurrence of duplications and, where the duplicons contain inverted repeats, the occurrence of inversions. A bias of ascertainment may explain the paucity of duplications and inversions observed so far. Usually the deletions cause the more severe phenotype. Nevertheless, 
several case reports have described the occurrence of duplications of several commonly deleted regions such as PraderWilli syndrome and VCFS. ${ }^{7,12}$ The recent findings of inversion polymorphisms at loci where duplicons are present such as in the WBS critical region, ${ }^{3} 8$ p commonly deleted region, ${ }^{5}$ and the Emery Dreifuss muscular dystrophy region $^{11}$ raise the expectation that inversions could be much more common than previously expected. Since large inverted sub-repeats are present within the VCFS duplicons, it is surprising that no inversion was detected within the VCFS common deleted region. Considering that inversion polymorphisms are likely to increase the susceptibility to chromosomal rearrangements, it could be expected that, if such polymorphisms exist, we would have detected them as they would be enriched in the chromosomes of parents transmitting a chromosome with the VCFS microdeletion. Several hypotheses can be put forward for the present observation: (A) The generation of inversions between inverted duplicons is a rare event and the common inversion polymorphisms that have been observed are the result of a single or only a few ancient inversion events. No such event has occurred in the VCFS region or, if such events have occurred, they did not become embedded in the human population. (B) Certain sequences are more prone to induce homologous recombination. Work from a number of groups has suggested that most human meiotic recombination is restricted to hot spots about $2 \mathrm{~kb}$ in length. Over $90 \%$ of recombination may be confined to these hot spots. $^{13}$ Sequences within the $10 \mathrm{~kb}$ VCFS inverted sub-repeats may be less prone to homologous recombination than other areas within the $200 \mathrm{~kb}$ replicon and therefore they do not mediate the inversions. (C) Selection acts against certain inversion polymorphisms. The inversion may in itself result in a rearrangement of a functional gene that is present within the duplicon. This has been shown to occur in Hunter syndrome and Hemophilia A. About $45 \%$ of severe hemophilia A males carry a large inversion on $\mathrm{Xq}$ which disrupts the factor VIII gene. ${ }^{1,14}$ About $20 \%$ of Hunter syndrome patients involve an inversion that disrupts the iduronate-2-sulfatase (IDS) gene, a

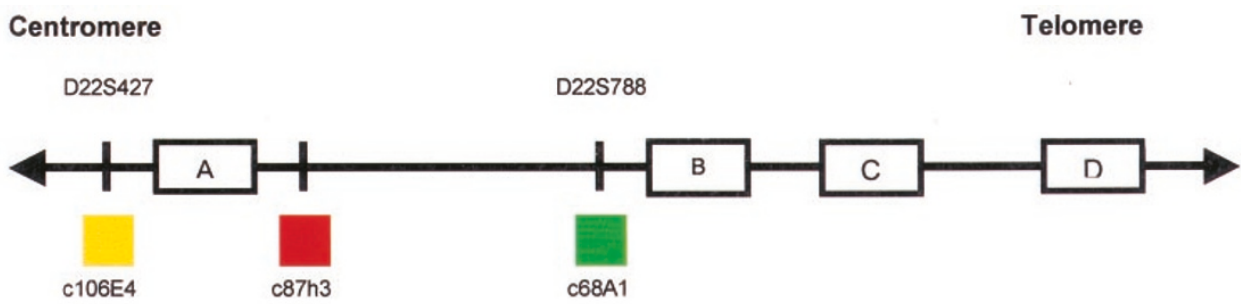

b

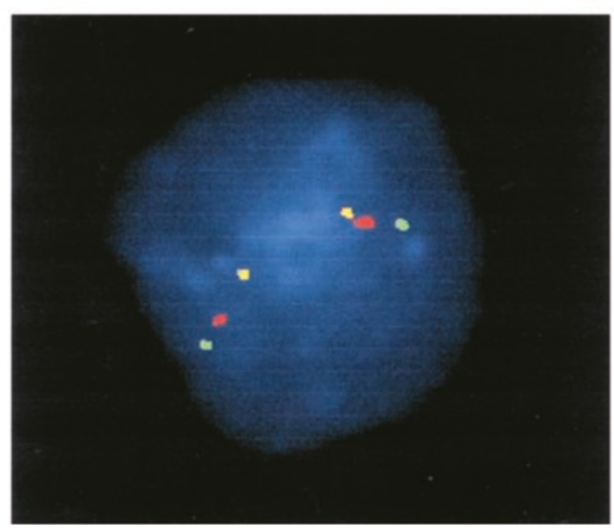

Figure 1 (a) Schematic map of the $3 \mathrm{Mb}$ commonly deleted region of chromosome 22q. This is not drawn to scale. The black boxes denote the position of the low copy repeat sequences flanking the 22q11 microdeletion region. [A] denotes the position of the proximal breakpoint, [D] the distal breakpoint of the $3 \mathrm{Mb}$ commonly deleted region and [B] and [C] the distal breakpoints of the less frequent, smaller deletions. The cosmid probes used for FISH analysis with their respective colour codes are indicated underneath. Adapted from Shaikh et al. (2000). (b) Dual colour FISH on interphase cells showing the typical alignment of the three cosmid probes on chromosome 22q11.2. 
caused by the homologous recombination between IDS and an IDS pseudogene $90 \mathrm{~kb}$ downstream. ${ }^{10}$ The inversion may also alter the chromatin structure and induce long-range effects on gene expression within the inverted region. Each of these hypotheses can be tested and further analysis of the sequences embedded between inverted repeat sequences will provide the answers.

In conclusion, this study did not identify an inversion polymorphism at 22q11. Nevertheless, the hypothesis that inversion polymorphisms are a major factor influencing susceptibility towards microdeletions remains intriguing. Therefore, it will be interesting to confirm the observations that an inversion polymorphism is a major susceptibility factor in the generation of microdeletions in the WBS and to investigate whether inversion polymorphisms exist within the duplicon boundaries surrounding the other common microdeletion syndromes.

\section{Acknowledgements}

Sponsored in part by the Belgian Foundation for Research in Pediatric Cardiology.

Gabriel Stefan Gebhardt Department of Obstetrics and Gynaecology, University of Stellenbosch, South Africa

Koenraad Devriendt, Reinhilde Thoelen, Ann Swillen, Elly Pijkels, Jean-Pierre Fryns, Joris R Vermeesch Center of Human Genetics, Herestraat 49, 3000 Leuven, Belgium

Marc Gewillig

Department of Paediatric Cardiology, University Hospital Gasthuisberg, University of Leuven, Leuven, Belgium

\section{References}

1 Lupski JR: Genomic disorders: structural features of the genome can lead to DNA rearrangements and human disease traits. Trends Genet 1998; 14: 417-422.

2 Ji Y, Eichler EE, Schwartz S, Nicholls RD: Structure of chromosomal duplicons and their role in mediating human genomic disorders. Genome Res 2000; 10: 597-610.

3 Osborne LR, Li M, Pober B et al: A 1.5 million-base pair inversion polymorphism in families with Williams-Beuren syndrome. Nat Genet 2001; 29: $321-325$.

4 Floridia G, Piantanida M, Minelli A et al: The same molecular mechanism at the maternal meiosis I produces mono- and dicentric 8p duplications. Am J Hum Genet 1996; 58: 785 - 796.

5 Giglio S, Broman KW, Matsumoto N et al: Olfactory receptor-gene clusters, genomic-inversion polymorphisms, and common chromosome rearrangements. Am J Hum Genet 2001; 68: 874-883.

6 Devriendt K, Swillen A, Fryns JP: Deletion in chromosome region 22q11 in a child with CHARGE association. Clin Genet 1998; 53: $408-410$.

7 Edelmann L, Pandita RK, Spiteri E et al: A common molecular basis for rearrangement disorders on chromosome 22q11. Hum Mol Genet 1999; 8: $1157-1167$.

8 Edelmann L, Pandita RK, Morrow BE: Low-copy repeats mediate the common $3-\mathrm{Mb}$ deletion in patients with velo-cardio-facial syndrome. Am J Hum Genet 1999; 64: 1076-1086.

9 Shaikh TH, Kurahashi H, Emanuel BS: Evolutionarily conserved low copy repeats (LCRs) in 22q11 mediate deletions, duplications, translocations, and genomic instability: an update and literature review. Genet Med 2001; 3: 6-13.

10 Bondeson ML, Dahl N, Malmgren $\mathrm{H}$ et al: Inversion of the IDS gene resulting from recombination with IDS-related sequences is a common cause of the Hunter syndrome. Hum Mol Genet 1995; 4: 615-621.

11 Small K, Iber J, Warren ST: Emerin deletion reveals a common Xchromosome inversion mediated by inverted repeats. Nat Genet JID - 9216904 1997; 16: 96-99.

12 Roberts SE, Dennis NR, Browne CE et al: Characterisation of interstitial duplications and triplications of chromosome 15q11-q13. Hum Genet 2002; 110: $227-234$.

13 Jeffreys AJ, Kauppi L, Neumann R: Intensely punctate meiotic recombination in the class II region of the major histocompatibility complex. Nat Genet 2001; 29: 217-222.

14 Lakich D, Kazazian HHJ, Antonarakis SE, Gitschier J: Inversions disrupting the factor VIII gene are a common cause of severe haemophilia A. Nat Genet 1993; 5: 236-241. 